\title{
Curcumin suppresses an endometrial cell inflammation through inhibition of SREBP-1
}

\author{
So Young Kim ${ }^{1 \#}$, Yi Yi Kyaw ${ }^{1 \#,}$ Mi So Seong1, Ki Hyung Kim² and JaeHun Cheong ${ }^{1 *}$ \\ ${ }^{1}$ Department of Obstetrics and Gynecology, Pusan National University School of Medicine, Medical Research Institute, Pusan National University Hospital, Korea \\ ${ }^{2}$ Department of Molecular Biology, College of Natural Sciences, Pusan National University, Busan 609-735, Korea; Department of Medicine, Pusan National \\ University, Busan 602-351, Korea \\ \#Equal contribution
}

\begin{abstract}
Curcumin is a natural polyphenolic compound extracted from the spice, turmeric and has been reported to evidence anti-inflammatory, antioxidant, and antiproliferative properties via the modulation of multiple cellular mechanisms. Endometriosis, the presence of ectopic endometrial tissue outside the uterine cavity, is a common disease affecting women during their reproductive years. In this study, we showed the effects of curcumin on endometrial cell inflammation. Curcumin inhibited expression of cyclooxygenase-2, which is a pivotal player in inflammatory processes, in the endometrium cells by using luciferase assays, real-time PCR and Western blotting analyses. Furthermore, curcumin exerted inhibitory effects via NF- $\mathrm{B}$-dependence in part. Decrease of inflammatory gene expression by curcumin in ovary cells was mediated by inhibition of transcription factor SREBP-1 and Akt signaling. In conclusion, the results indicate that curcumin may be potentially useful as novel anti-inflammatory reagents when administered in endometriosis.
\end{abstract}

\section{Introduction}

There is general agreement that endometriosis is a chronic pelvic inflammatory process, characterized by enhanced numbers of activated peritoneal immune cells and pro-inflammatory factors $[1,2]$. In particular, increased concentrations of prostaglandins (PGs) and leukotrienes have been found in the peritoneal fluid of endometriosis patients [3]. These are the major constituents of a group of biologically active oxygenated fatty acids known as eicosanoids and have been implicated in various inflammatory diseases. In endometriosis, they appear to play an important role in disease-associated pain, essentially treated with non-steroidal anti-inflammatory drugs [4]. These inflammatory mediators, particularly PGs, may also be directly involved in the pathogenesis of endometriosis, as recent in vitro studies have demonstrated that improved synthesis is involved in enhancing proliferation while inhibiting apoptosis, increasing both angiogenesis and immunosuppression [5]. The cyclooxygenase (COX) pathway leads to the formation of PGs.

Curcumin is a natural polyphenolic compound extracted from the spice, turmeric and has been reported to evidence anti-inflammatory, antioxidant, and anti-proliferative properties via the modulation of multiple cellular [6]. However, it remains to be determined whether curcumin affects in the process of endometriosis.

The evolution of hepatic inflammation is controlled by specific transcriptional regulators, some of which are well known in the context of cholesterol-inducible inflammation (SREBPs, NF- $\kappa \mathrm{B}$, AP-1, C/EBPs) [7]. Interestingly, some of these factors may also represent molecular links between lipid/cholesterol metabolism and inflammation. Considering that SREBP-1 can promote inflammation and be regulated by hypoxia, we showed that low cellular oxygen tension in endometriotic stromal cells up-regulated SREBP1 expression to increase the inflammation of ectopic endometrial cells. In addition, we showed that curcumin at concentrations that do not affect cell viability reduced hypoxia-induced COX-2 and SREBP-1 expression to a significant degree. We reported here for the first time the antiendometriotic property of curcumin via COX-2 repression pathway that may lead to new therapeutic intervention.

\section{Materials and methods}

\section{Subjects}

Ten women (ages, 26-39 yr; mean age, 31.4 \pm 3.7 ) with endometriosis (I/II) were enrolled in this study. These women treated for infertility and/or pelvic pain, were found to have endometriosis during laparoscopy, and had not received any anti-inflammatory, or any other hormonal treatment during a period of at least 3 months before surgery. All patients enrolled in this study were in proliferative phases of the menstrual cycle. Specimens were obtained from peritoneum or ovarian surface. Control tissues were obtained from ten women. The collection of uterine endometrium was carried out by endometrial sampling procedure using sterile, disposable endometrial suction curettes (Z-sampler, Zinnanti, Chatsworth, CA, USA). Endometriotic biopsies were immediately placed at $4^{\circ} \mathrm{C}$ in sterile Hanks' balanced salt solution (HBSS) containing $100 \mathrm{U} / \mathrm{mL}$ penicillin, $100 \mathrm{mg} / \mathrm{mL}$ streptomycin, and $0.25 \mathrm{mg} / \mathrm{mL}$ amphotericin. All cases were diagnosed and staged as

${ }^{\star}$ Correspondence to: JaeHun Cheong, Department of Molecular Biology, Pusan National University, Busan, 609-735, Korea, Tel: 82-51-510-2277; Fax: 82-51513-9258; E-mail: molecule85@pusan.ac.kr

Key words: curcumin, COX-2, endometriosis, SREBP-1

Received: September 20, 2019; Accepted: October 09, 2019; Published: October 16,2019 
endometriosis according to the revised classification of The American Society of Reproductive Medicine and confirmed by histopathological characterizations. Written informed consent was obtained from all of the patients and the Institutional Review Board at Pusan National University Hospital approved this protocol.

\section{Endometrial cell culture}

The tissues were treated with $0.25 \%$ collagenase type I in DMEM at $37^{\circ} \mathrm{C}$ for $30 \mathrm{~min}$ in a shaking water bath and subsequently the cells were passed through 100- and 70- $\mu \mathrm{m}$ sieve (BD Falcon, Bedford, MA). After sedimentation, the supernatant was transferred to a new tube and cells were collected by centrifugation. The cells were maintained and proliferated in DMEM supplementing with $10 \%$ fetal calf serum (FCS), in a humidified atmosphere of $5 \% \mathrm{CO}_{2}$ at $37^{\circ} \mathrm{C}$. The stromal cellrich supernatant was placed in a culture flask and cells were allowed to adhere for 20 minutes then washed with medium. Adherent stromal cells were cultured as monolayers in flasks with DMEM/F-12 (1:1) (Sigma) containing antibiotics/antimycotics, $5 \mu \mathrm{g} / \mathrm{mL}$ insulin (Sigma), and 10\% FCS: normal endometrial stromal cell (ES), stromal cell of ovarian red lesion (ORS), stromal cell of ovarian black lesion (OBS). Purity of cultures was determined morphologically after H\&E staining. Stromal cells were fusiform, but more rounded than fibroblasts. Cells were also evaluated immunohistochemically using monoclonal antibodies to human cytokeratin, vimentin, von Willebrand factor, and CD45.

\section{Cell line culture and Treatments}

The A2780 cells were maintained in Dulbecco's modified Eagle's medium (DMEM) containing $10 \%$ fetal bovine serum (FBS) at $37^{\circ} \mathrm{C}$ in a humid atmosphere of $5 \% \mathrm{CO}_{2}$. A2780 and endometrial cells were plated at $70 \%-80 \%$ confluence and treated with various concentrations of curcumin or vehicle controls and incubated for 24 hours at $37^{\circ} \mathrm{C}$.

\section{Western blotting and nuclear/cytosolic fractions analysis}

A2780 cells were lysed in a radio-immunoprecipitation assay (RIPA) buffer containing $150 \mathrm{mmol} / \mathrm{L} \mathrm{NaCl}, 50 \mathrm{mmol} / \mathrm{L}$ Tris-Cl, $\mathrm{pH}$ 7.4, $1 \mathrm{mmol} / \mathrm{L}$ ethylenediaminetetraacetic acid (EDTA), 1\% Nonidet $\mathrm{P}-40,0.25 \% \mathrm{Na}$ deoxycholate, $1 \mathrm{mmol} / \mathrm{L}$ phenylmethylsulfonyl fluoride (PMSF), and protease inhibitors for 20 minutes on ice, after which the whole-cell lysates were acquired via subsequent centrifugation. The protein concentration was determined via a Bradford assay (Bio-Rad, Hercules, CA). Fifty micrograms of protein from the cell lysates was subjected to $8 \%$ sodium dodecyl sulfate-polyacrylamide gel electrophoresis (SDS-PAGE) and transferred to a polyvinylidene difluoride (PVDF) membrane (Millipore, Billerica, MA) via semidry electroblotting. The membranes were then incubated for $1 \mathrm{~h}$ at room temperature with rabbit CDX1 antibody and anti-actin antibody (A2066, Sigma) in Tris-buffered saline Tween-20 supplemented with $1 \%$ nonfat dry milk. The bands were detected using an enhanced chemiluminescence system (Amersham, Piscataway, NJ). The protein bands were quantified and normalized relative to the loading control band with ImageJ version 1.35d (National Institutes of Health Image software, Bethesda, MD).

To prepare cytosolic and nuclear lysates, cells were lysed in buffer A (10 mM HEPES, pH 7.9, $1.5 \mathrm{mM} \mathrm{MgCl}_{2}, 10 \mathrm{mM} \mathrm{KCl}, 1 \mathrm{mM} \mathrm{DTT}, 0.5 \%$ NP-40, 1mM PMSF, protease inhibitors) and incubated for $10 \mathrm{~min}$ on ice. The supernatants (cytosolic lysates) were collected by centrifugation (6000rpm) at $4^{\circ} \mathrm{C}$ for $5 \mathrm{~min}$. The nuclear pellets were then washed with ice-cold PBS to avoid contamination of cytosolic proteins and lysed in buffer B (10 mM HEPES, pH 7.9, $1.5 \mathrm{mM} \mathrm{MgCl}, 10 \mathrm{mM} \mathrm{KCl,} \mathrm{25 \%}$
Glycerol, 420 mM NaCl, 0.2 mM EDTA, 1 mM DTT, 0.5\% NP-40, 1mM PMSF, protease inhibitors). After incubation on ice for $25 \mathrm{~min}$, the supernatants (nuclear lysates) were collected by centrifugation (12000 rpm) at $4^{\circ} \mathrm{C}$ for $5 \mathrm{~min}$.

\section{siRNA design and siRNA transfection}

RNA oligonucleotides were synthesized by Bioneer (Daejeon, Republic of Korea). The sequences of siRNA targeting human p65 were sence, 5'-gauugaggagaaacguaaa-3' and antisense, $5^{\prime}$-uuuacguuucuccucaauc- $3^{\prime}$. The scramble control siRNA sequences were sense, 5'-ccuacgccaccaauuucgu-3' and antisense, 5 '-acgaaauugguggcguagg- 3 '. The cells were transfected with siRNA using HiPerFect (Qiagen) according to the instructions of the manufacturer.

\section{Real-time PCR analysis}

Total RNA from curcumin treated A2780 cells was prepared using TRIzol reagent (Invitrogen) by following the manufacturer's instructions. cDNA was used as a template for real-time PCR using gene-specific primers: GAPDH, 5'-gtggtctcctctgacttcaac-3' (forward) and 5'-tctcttcctcttgtgctcttg-3' (reverse). Real-time PCR was carried out in a LightCycler (Roche) using a LightCycler Fast Start DNA Master SYBR Green I kit (Roche).

\section{Transient transfection and luciferase reporter assay}

Plasmid transfection was conducted using PolyFect (Qiagen) in accordance with the manufacturer's instructions. The pcDNA3.1/HisC empty vector was added to the transfections in order to achieve the same total amount of plasmid DNA per transfection. The cells were lysed in cell culture lysis buffer (Promega). The luciferase activity was evaluated using an analytical luminescence luminometer in accordance with the manufacturer's instructions. Luciferase activity was normalized for transfection efficiency using the corresponding $\square$-galactosidase activity. The assays were performed in three independent experiments, and the results were expressed as the mean \pm S.D as percentages of the controls.

\section{Cell viability assay}

Cell viability was determined via MTT assay. In brief, A2780 cells were seeded on 24-well culture plates and treated with the drug as indicated in the Figure Legends for 24 hours. The media were removed and replaced with $100 \mu \mathrm{l}$ of a $5 \mathrm{mg} / \mathrm{ml}$ tetrazolium bromide solution (3-[4,5-dimethylthiazol-2-yl]-2,5-diphenyltetrazolium bromide; Sigma) for $1 \mathrm{~h}$ at $37^{\circ} \mathrm{C}$. The addition of $200 \mu \mathrm{l}$ of DMSO resulted in color development. The supernatants were then removed and placed into 96-well trays for analysis. Optical density values were determined at $590 \mathrm{~nm}$.

\section{Statistical analysis}

Statistical analyses were conducted via unpaired or paired $t$ tests as appropriate. All data were expressed as the means \pm SD. $P$ values of $<$ 0.05 were considered significant.

\section{Results}

\section{Curcumin represses hypoxia-mediated COX-2 and SREBP-1 expression in endometrial cells from human patient lesions}

In previous result, we presented that hypoxia increases proinflammatory proteins COX-2 expression in endometrial cells of human patient lesions and cultured ovary cells [8]. Based on other reports, curcumin decreases expression of various proinflammatory regulators in several tissues [9-11]. In order to examine whether 
curcumin affects expression of proinflammatory transcriptional regulator, NF- $\kappa \mathrm{B}$ and SREBP-1 in endometriosis, we evaluated protein levels of NF- $\kappa$ B p65 and mature form of SREBP-1 by curcumin treatment in the presence or absence of hypoxia stimuli in endometrial lesion cells. As shown in Figure 1, while hypoxia increased the protein expression of COX2, p65 subunit of NF- $\mathrm{kB}$, and mature form of SREBP1 in the normal endometrial stromal cell (ES), stromal cell of ovarian red lesion (ORS), stromal cell of ovarian black lesion (OBS), curcumin significantly decreased expression of COX-2 and mature form of SREBP-1 in endometrial cells, but not p65 protein of NF- $\kappa$ B. These results indicate that curcumin decreases induction of a proinflammatory mediator by suppression of SREBP1 activation.

\section{Curcumin inhibits COX-2 expression via NF- $\mathrm{kB}$ repression in part}

In an effort to gain insight into the molecular mechanism by which curcumin suppresses COX-2 expression, we then analyzed the signaling pathways involved in curcumin-inhibited COX-2 expression. In previous reports, NF- $\mathrm{kB}$ increases COX-2 expression at the transcriptional level dependent on its promoter binding. To evaluate the effect of NF- $\mathrm{KB}$ pathway on curcumin-inhibited COX-2 expression, p65 siRNA and Bay11-7082 (a specific inhibitor of NF- $\mathrm{KB}$ pathway) were exploited. In contrast to our expectations, p65 siRNA and Bay11-7082 had no effect on COX-2 expression in SKOV3 cells (Figures $2 \mathrm{~A}$ and $2 \mathrm{~B}$ ). However, in the experiments using another ovary cell line A2780, COX-2 promoter activation was decreased by both of p65 siRNA and Bay11-7082 (Figures 2A and 2B). Furthermore, curcumin also inhibited the nuclear localization of p65 (Figure 2C). Taken together, NF- $\kappa B$ activation partly mediated the transcriptional expression of COX-2 in ovary cells in a cell context-dependent manner, suggesting that another transcription factors might involved in COX2 expression and curcumin-mediated suppression of COX-2 in ovary cells and endometriosis.

\section{Curcumin suppresses COX-2 and SREBP-1 promoter activation}

In order to further know the inhibitory effects of curcumin on the induction of proinflammatory regulators, COX-2 enzyme and SREBP-1 transcriptional regulator, human ovary cell line A2780 cells were transfected with promoter reporter genes of COX-2 and SREBP-1 in the presence or absence of curcumin treatment. The promoter repression of COX-2 and SREBP- 1 in the A2780 cells demonstrated that curcumin inhibits activation of promoter expression of COX-2 and SREBP-1
(Figures $3 \mathrm{~A}$ and $3 \mathrm{~B}$ ). The MTT assay showed that the curcumin concentrations chosen were subcytotoxic (Figures $3 \mathrm{~A}$ and $3 \mathrm{~B}$ ). These data indicate that the inhibitory effects on COX-2 and SREBP-1 expression exerted by curcumin are not attributable to cytotoxicity or to an artificial effect.

In an effort to confirm these inhibitory effects on the SREBP-1 gene expression by curcumin, we further confirmed whether curcumin affects the SREBP-1 RNA and protein level. SREBP-1 RNA level was quantified by real-time RT-PCR and protein level was assayed by Western blots with the anti-SREBP-1 antibody. As shown in Figure 3C, curcumin-treated cells expressed lower levels of the SREBP-1 RNA as compared with the control vehicle-treated A2780 cells. Also, we showed that curcumin decreased the protein level of SREBP-1 (Figure 3C). These data correlate well with the luciferase activity of A2780 ovary cells.

To further examine whether an increase of the transactivation of the COX-2 promoter by SREBP-1 results from SREBP-1 recruitment on the responsive DNA element of the COX-2 promoter, it was applied to the chromatin immunoprecipitaion (Chip) assay. Isolated DNA was subjected to PCR both before (Input) and after Chip using primer sets designed to amplify the region with the COX-2 promoter harboring the SREBP-1 binding site. Chip assay demonstrated that SREBP-1 can form a transcriptional complex on COX-2 promoter ( -433 to -422$)$ (Figure $3 \mathrm{D})$. However, there is no amplified PCR products at the pcDNA/HA transfection. The result suggests that SREBP-1 increases the COX-2 expression at the transcriptional level through direct DNA binding and curcumin inhibits DNA binding activity and transcriptional activation of SREBP-1.

\section{Curcumin inhibits COX-2 expression dependent on PI3K/ Akt and SREBP-1 pathway}

In an effort to gain insight into the molecular mechanism by which curcumin suppresses COX-2 expression, we then analyzed the signaling pathways involved in curcumin-inhibited SREBP-1 expression. Several signaling pathways and transcription factors including AP-1, mitogenactivated protein kinases (MAPKs), and cell cycle machinery have been suggested as the targets of curcumin. We examined the effect of several pathways on SREBP-1 expression using treatments of specific kinase inhibitors or transfection of plasmid for specific kinases, including ERK, JNK, p38, Akt, and PKA. In previous our study, we identified that curcumin inhibited the PI3K/Akt signaling activation [12]. Consistent with this finding, among of many signaling pathways, transfection
ES

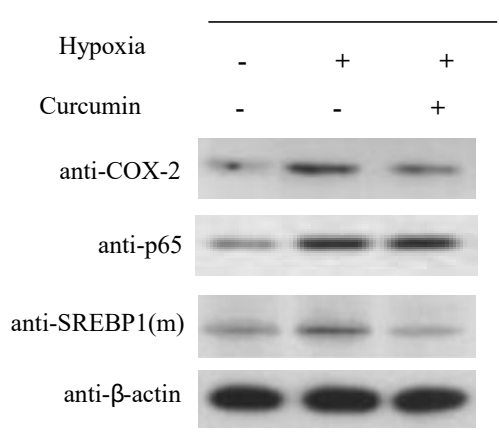

ORS

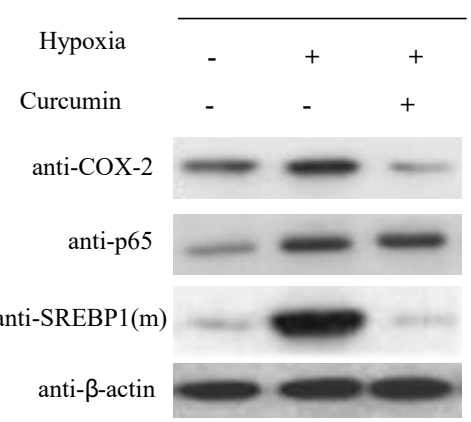

OBS

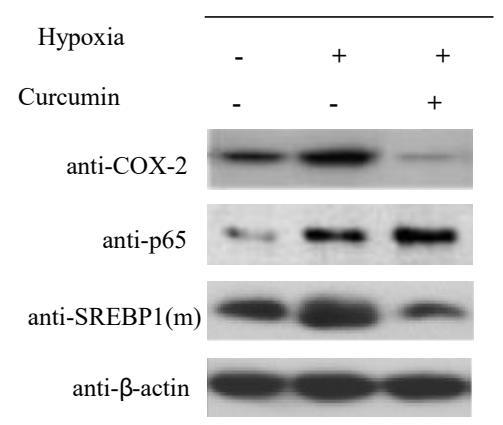

Figure 1. Curcumin represses hypoxia-mediated COX-2 expression in endometrial cells from human patient lesions. Endometrial ovarian stromal cells of human patients were treated hypoxia ( $2 \%$ ) and curcumin. Total protein was prepared from the cells and then, the protein levels of COX-2, NF- $\mathrm{KB}$ p 65 and mature form of SREBP-1 were detected by Western blotting with $\beta$-actin as loading control. ES; normal endometrial stromal cell, ORS; stromal cell of ovarian red lesion, OBS; stromal cell of ovarian black lesion 
$\mathbf{A}$

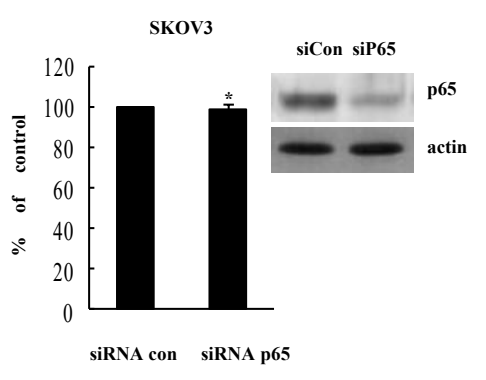

B

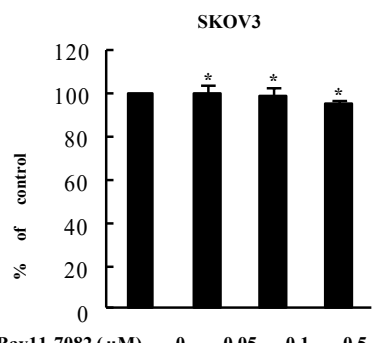

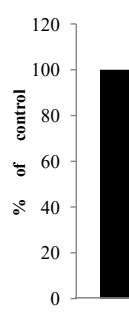

Bay11-7082 $(\mu \mathrm{M})$

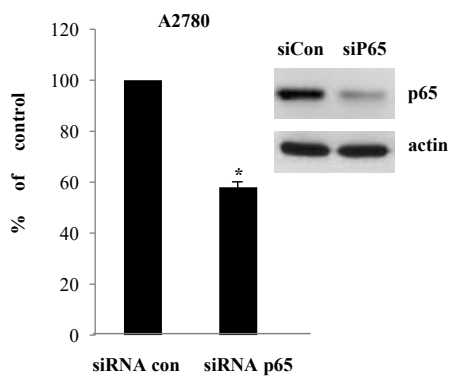

C

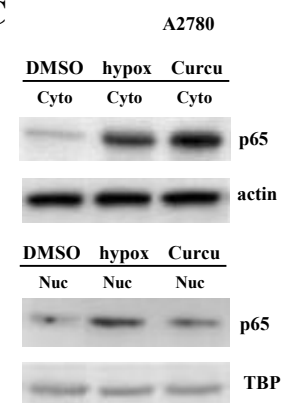

Figure 2. Inhibition of COX-2 by curcumin is not dependent on NF- $\kappa \mathrm{B}$ activation (A) Knock-down of NF- $\mathrm{kB}$ p65 expression does not change COX-2 induction in SKOV3 and A2780 cells. The indicated ells were transiently transfected with control siRNA and NF- $\kappa B$ p 65 -specific siRNA. RT-PCR of p65 expression was done for confirming reduced p65 level. $* P<0.01$ compared

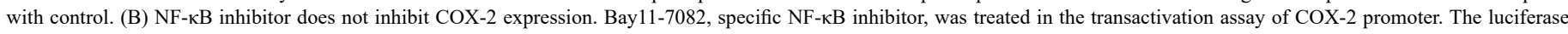
activity was determined at $24 \mathrm{~h}$ of treatment. ${ }^{*} P<0.01$ compared with control. (C) Curcumin inhibits NF- $\mathrm{KB}$ nuclear locatization. After treatment of curcumin and vehicle (DMSO), cells

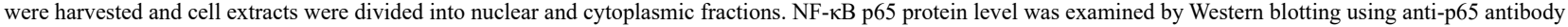

A
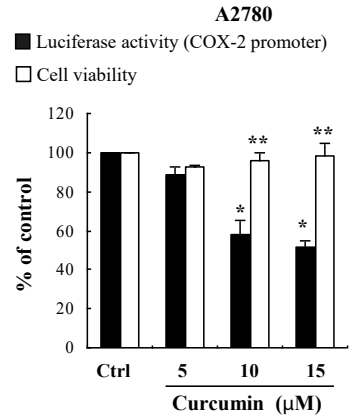

B
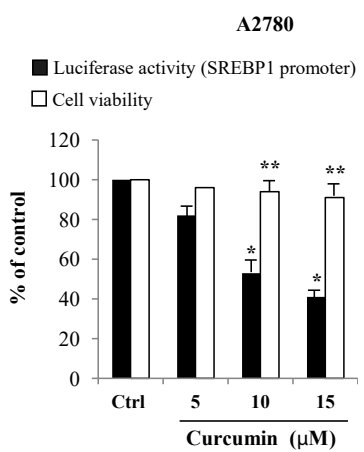

C

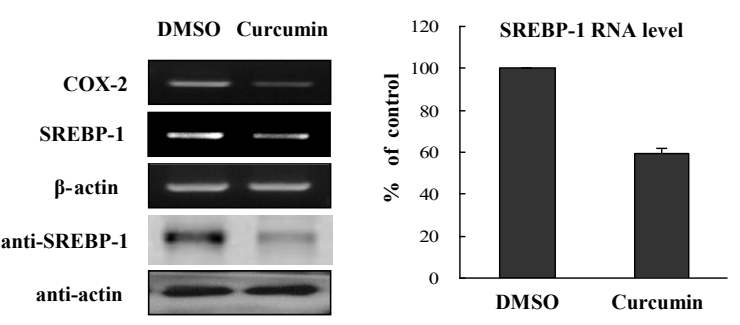

D

COX-2 -433/-422

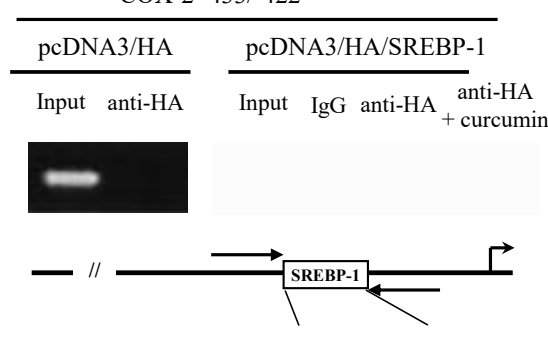

-433 ATCAGTCCAC -422

Figure 3. Curcumin suppress COX-2 and SREBP-1 expression at the transcriptional level (A) Curcumin inhibits the promoter activity of COX-2 gene. For the luciferase assay, the A2780 cells were transfected with COX-2 promoter reporter plasmid in the presence of the indicated curcumin concentrations. After $24 \mathrm{~h}$ of curcumin treatment, MTT assays were conducted. The data are expressed as the means \pm S.D $(\mathrm{n}=3$ ). $* P<0.01$ compared with control. $* * P<0.05$ compared with control. (B) Curcumin decreases the promoter activity of SREBP-1. (C) Curcumin decreases mRNA and protein expression of COX-2 and SREBP-1 in ovary cells. After curcumin treatment, cells were applied for RT-PCR and Western blotting. Actin was used for a loading control. (D) The chromatin immunoprecipitation (Chip) assay. Empty vector pcDNA3/HA and pcDNA3/HA/SREBP-1 expression vector were transfected into A2780 cells in the presence or absence of curcumin treatment. Following formaldehyde cross-linking, soluble chromatin was prepared. After immunoprecipitation with HA antibody, precipitated DNAs were used in PCR analysis. The input lane shows the starting chromatin extracts 

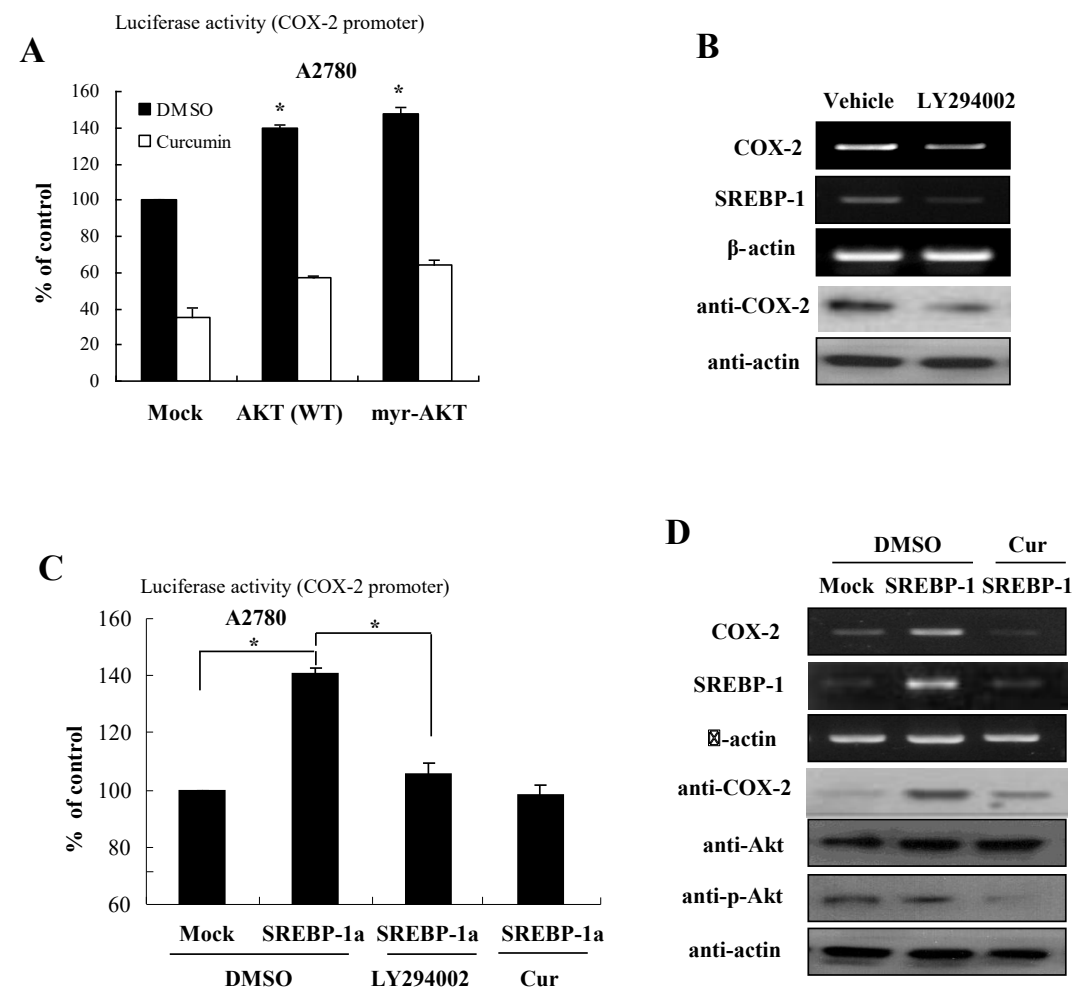

Figure 4. Curcumin inhibits COX-2 expression through repression of the Akt-SREBP1 signaling activation (A) Akt signaling is involved in curcumin-directed COX-2 inhibition. Expression constructs of Akt and myr-Akt were transfected into A2780 cells and the cells were applied to luciferase reporter assay in the presence or absence of curcumin treatment. (B) Akt inhibitor decreases mRNA and protein expression of SREBP-1 in ovary cells. After treatment of LY294002, specific inhibitor of Akt, cells were applied to analyze mRNA and protein expression by RT-PCR and Western blotting, respectively. (C) Curcumin inhibits SREBP-1-directed COX-2 promoter activation. A2780 cells were transfected with SREBP-1 expression plasmid and COX-2 promoter reporter in the presence of curcumin or LY294002. The cells were analyzed for luciferase assay. (D) Curcumin inhibits COX-2 expression by suppression of Akt phosphorylation. A2780 cells were transfected with SREBP-1 in the presence or absence of curcumin treatment. After harvesting, cells were applied for RT-PCR and Western blotting with the indicated antibodies

of plasmid for wild type (WT)-Akt or constitutive active (myr)-Akt significantly augmented the luciferase activities of SREBP-1 gene (Figure 4A). In order to identify whether the Akt signaling is involved in curcumin-directed COX-2 inhibition, we examined the mRNA and protein expression of COX-2 in the presence or absence of LY294002, which is an inhibitor of PI3K/Akt. As shown in Figure 4B, LY294002 suppressed COX-2 expression in the mRNA and protein level. As predicted, SREBP-1 was also decreased with LY294002 treatment (Figure 4B).

To confirm these effects on the COX-2 expression regulated by PI3K/Akt signaling, we examined the luciferase activity of COX-2 promoter with SREBP-1 overexpression in the presence or absence of LY294002 and curcumin. The results of Figure 4C showed that while forced expression increased COX-2 promoter activation, both treatements of LY294002 and curcumin suppressed the SREBP-1 mediated activation of COX- 2 promoter. In addition, we further confirmed that the COX-2 RNA and protein levels were sensitive to LY294002 and curcumin decreased Akt phosphorylation and subsequent SREBP-1 expression, finally leading to COX-2 inhibition (Figure 4D). Taken together, these results suggest that curcumin suppresses COX-2 expression via inhibition of PI3K/Akt and SREBP-1 activation pathway in ovary cells.

\section{Discussion}

Endometriosis is more susceptible to neoplasia induction. Understanding the steps leading to endometriosis is crucial for the identification of early prognostic or diagnostic markers of tumor development. Chronic inflammation and associated endometriosis are fairly common precancerous conditions for ovarian cancer.

Based on the findings of increased expression of aromatase P450 in the endometriotic tissue, some investigators used aromatase inhibitors (Als) to treat pain symptoms in patients with endometriosis $[13,14]$. However, other researcher proposed that Als need to be investigated further in well-designed studies to confirm the hypothetical impact on endometriotic lesions, since there have been no strong evidences favoring the efficacy or benetif of Als compared to other hormonal drugs in currently available clinical trials $[15,16]$.

Since mounting evidence showed that altered immune function plays a crucial role in the pathogenesis and pathophysiology of endometriosis, several investigators suggested that modulating the inflammation could be an alternative approach for treatment of endometriosis [17]. Anti-tumor necriosis factor-alpha (anti-TNF- $\alpha$ ) was evaluated the effectiveness and safety in the management of pelvic pain associated with endomertiosis. However, results showed no evidence of improvement of pain score or reduced use of pain killers after treating by infliximab, one of the known anti-TNF- $\alpha$ drugs [18]. Here we show that curcumin can be used a newer drug for decreasing then inflammation in the process of endometriosis.

It has been shown that curcumin regulates diverse molecular targets implicated in inflammation. Specifically, curcumin inhibits inflammatory cytokines such as TNF- $\alpha$, IL-1, -2, -6, -8, -12, MAPK, and JNK, as well as suppresses the inducible nitric oxide synthase, COX-2, and lipoxygenase in a variety of cancer cells [10]. Furthermore, 
curcumin suppresses IL- $1 \beta$-induced NF- $\kappa B$ activation and nuclear translocation as well as IL-1 $\alpha$ induced PI3K/Akt activation [19]. The results of Figure 4 showed that Akt activation is required to modulate COX-2 expression in ovary cells and curcumin inhibits the Akt-directed COX-2 induction. Curcumin decreases inflammation in the adipose liver steatosis through the phosphorylation of the signal transducer and activator of transcription 3 (STAT3), as well as through downregulation of suppressor of cytokine signaling 3 (SOCS3) and SREBP-1 in livers of obese mice $[20,21]$. In this study, curcumin suppressed the PI3K/Akt signaling activation and subsequent SREBP-1 expression, which up-regulates COX-2 expression, in the endometrial cells and A2780 ovary cells.

Moreover, the present observations suggest that the identification of the factors required for the hypoxia-induced inflammation in the process of endometriosis and the clarification of the molecular mechanisms responsible for an early phenotypic change in endometriosis may facilitate the development of therapeutic interventions.

\section{References}

1. Riccio LDGC, Santulli P, Marcellin L, Abrão MS, Batteux F, et al. (2018) Immunology of endometriosis. Best Pract Res Clin Obstet Gynaecol 50: 39-49. [Crossref]

2. Vercellini P, Somigliana E, Viganò P, Abbiati A, Daguati R, et al. (2008) Endometriosis: current and future medical therapies. Best Pract Res Clin Obstet Gynaecol 22: 275-306. [Crossref]

3. Jabbour HN, Sales KJ, Catalano RD, Norman JE (2009) Inflammatory pathways in female reproductive health and disease. Reproduction 138: 903-919.

4. Brown J, Crawford TJ, Allen C, Hopewell S, Prentice A (2017) Non-steroidal antiinflammatory drugs for pain in women with endometriosis. Cochrane Database Syst Rev 23: CD004753.

5. Wu MH, Shoji Y, Chuang PC, Tsai SJ (2007) Endometriosis: disease pathophysiology and the role of prostaglandins. Expert Rev Mol Med 9: 1-20. [Crossref]

6. Aggarwal BB, Shishodia S (2006) Molecular targets of dietary agents for prevention and therapy of cancer. Biochem Pharmacol 71: 1397-1421.

7. Kleemann R, Verschuren L, van Erk MJ, Nikolsky Y, Cnubben NH, et al. (2007) Atherosclerosis and liver inflammation induced by increased dietary cholesterol intake: a combined transcriptomics and metabolomics analysis. Genome Biol 8: R200. [Crossref]
8. Kim KH, Kim HY, Kim HH, Lee KS, Cheong JH (2011) Hypoxia induces expression of COX-2 through the homeodomain transcription factor CDX1 and orphan nuclear receptor SHP in human endometrial cells. Mol Hum Reprod 17: 710-719.

9. Norris L, Karmokar A, Howells L, Steward WP, Gescher A, et al. (2013) The role of cancer stem cells in the anti-carcinogenicity of curcumin. Mol Nutr Food Res 57: 1630-1637. [Crossref]

10. Rahman I, Biswas SK, Kirkham PA (2006) Regulation of inflammation and redox signaling by dietary polyphenols. Biochem Pharmacol 72: 1439-1452.

11. Duvoix A, Blasius R, Delhalle S, Schnekenburger M, Morceau F, et al. (2005) Chemopreventive and therapeutic effects of curcumin. Cancer Lett 223: 181-190. [Crossref]

12. Kim K, Kim KH, Kim HY, Cho HK, Sakamoto N, et al. (2010) Curcumin inhibits hepatitis C virus replication via suppressing the Akt-SREBP-1 pathway. FEBS Lett 19: 707-712.

13. Słopień R, Męczekalski B (2016) Aromatase inhibitors in the treatment of endometriosis. Prz Menopauzalny 15: 43-47. [Crossref]

14. Olivares C, Bilotas M, Buquet R, Borghi M, Sueldo C, et al. (2008) Effects of a selective cyclooxygenase- 2 inhibitor on endometrial epithelial cells from patients with endometriosis. Hum Reprod 23: 2701-2708.

15. Ferrero S, Gillott DJ, Venturini PL, Remorgida V (2011) Use of aromatase inhibitors to treat endometriosis-related pain symptoms: a systematic review. Reprod Biol Endocrinol 9: 89. [Crossref]

16. Colette S, Donnez J (2011) Are aromatase inhibitors effective in endometriosis treatment? Expert Opin Investig Drugs 20: 917-931. [Crossref]

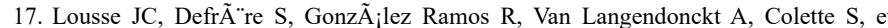
al. (2009) Involvement of iron, nuclear factor-kappa B (NF- B) and prostaglandins in the pathogenesis of peritoneal endometriosis-associated inflammation: a review. $J$ Endometr 1: 19-29.

18. Lv D, Song H, Shi G (2010) Anti-TNF-alpha treatment for pelvic pain associated with endometriosis. Cochrane Database Syst Rev 3: CD008088. [Crossref]

19. Buhrmann C, Mobasheri A, Busch F, Aldinger C, Stahlmann R (2011) Curcumin modulates nuclear factor kappaB (NF-kappaB)-mediated inflammation in human tenocytes in vitro: role of the phosphatidylinositol 3-kinase/Akt pathway. J Biol Chem 286: 28556-28566.

20. Jurenka JS (2009) Anti-inflammatory properties of curcumin, a major constituent of Curcuma longa: a review of preclinical and clinical research. Altern Med Rev 14: 141153.

21. Kuo JJ, Chang HH, Tsai TH, Lee TY (2012) Positive effect of curcumin on Inflammation and mitochondrial dysfunction in obese mice with liver steatosis. Int J Mol Med 30: 673-679.

Copyright: C2019 Kim SY. This is an open-access article distributed under the terms of the Creative Commons Attribution License, which permits unrestricted use, distribution, and reproduction in any medium, provided the original author and source are credited. 